\title{
1 Current trends and future directions in koala chlamydial disease \\ research
}

4 Running head: Koala chlamydial disease research systematic review McCallum $^{1}$

${ }^{1}$ Environmental Futures Research Institute and School of Environment, Griffith University, Nathan, 10 Queensland 4111, Australia.

$11{ }^{2}$ Koala Ecology Group, The University of Queensland, St Lucia, Queensland 4072, Australia.

$12{ }^{3}$ Dreamworld Wildlife Foundation, Dreamworld, Parkway, Coomera, Queensland 4209, Australia.

$14{ }^{*}$ Corresponding author

15 Current address: Griffith Wildlife Disease Ecology Group, Environmental Futures Research

16 Institute, School of Environment, Griffith University, Nathan, Queensland, 4111, Australia.

17 Tel: (+61 4) 02255204

18 Email: l.grogan@griffith.edu.au

19

20 Keywords: declines, koala, Phascolarctos cinereus, conservation, extinction, population,

21 demography, disease, Chlamydia

22

23 


\section{ABSTRACT}

26 Infectious diseases can be key threatening processes for biodiversity conservation. However,

27 establishing the relative importance of disease (among other threatening processes) as a driver of

28 species declines can be challenging. Bias in the directions that a research field may take as it

29 develops - due to factors such as conservation policy, funding, public perception, and available

30 expertise and technology - may exacerbate this difficulty. Chlamydiosis (infection with bacteria in

31 Family Chlamydiaceae) is an example of an infectious disease with a poorly understood role in

32 koala (Phascolarctos cinereus) population dynamics. The arboreal folivorous koala is an

33 internationally recognized iconic species of high conservation, sociocultural and economic value.

34 To date, no studies have quantitatively examined the breadth and scope of research related to koala

35 chlamydiosis, nor systematically identified the current research gaps. We systematically and

36 quantitatively reviewed a comprehensive database of literature related to koala chlamydiosis,

37 classified and examined the main foci of the research, and evaluated research gaps with the goal of

38 assisting policy planning for funding further koala chlamydiosis research. We examined published

39 literature with regards to journal category, authorship, funding, spatiotemporal scope, study foci and

40 type, chlamydial species examined, methodological design and overall findings. Among the 117

41 peer-reviewed papers published between 1970 and 2016 that fit our criteria, the most striking

42 finding was the relative lack of population-level disease studies within the last two decades to

43 examine mechanisms of chlamydial infection dynamics. This research gap is of particular concern

44 given the potential role of Chlamydia in koala population declines, and the recent dramatic changes

45 in our understanding of pathogen phylogeny and improved diagnostic approaches. Our results

46 demonstrate a pressing need for future in situ comprehensive longitudinal population-level studies

47 from diverse geographic regions. These studies must utilize up-to-date diagnostic methods capable

48 of distinguishing chlamydial species and strains to elucidate the role of chlamydial infection in 
49 koala population declines and the underlying mechanisms involved. They should also employ

50 rigorous epidemiological methodologies and evaluate co-infection, habitat, climatic and

51 demographic data. Our findings suggest some key research gaps concerning koala chlamydiosis,

52 and are hence important for guiding future research into koala chlamydiosis and conservation. 


\section{INTRODUCTION}

56 Infectious diseases are increasingly recognized as key threatening processes for the conservation of

57 biodiversity (Daszak 2000; Smith et al. 2009; Tompkins et al. 2015). Diseases such as

58 chytridiomycosis in amphibians, West Nile Virus in birds, and white nose syndrome in bats have

59 exerted devastating effects on wildlife species around the globe (George et al. 2015; Skerratt et al.

60 2007; Thogmartin et al. 2012). Microorganisms and parasites are, however, ubiquitous among hosts

61 and in the environment, and many recognized infectious agents persist in reservoirs in the absence

62 of clinical disease (Haydon et al. 2002). Thus, determining the specific role of infectious agents in

63 host population declines can be difficult, particularly if their effects are not readily detected in the

64 form of mass mortalities and/or overt disease (Scheele et al. 2016; Smith et al. 2009).

65

66 Chlamydiosis (associated with infection by bacteria in Family Chlamydiaceae) is an example of an

67 infectious disease thought to be endemic and ubiquitous in koala populations (Phascolarctos

68 cinereus, Goldfuss), however, it has a poorly understood role in host population declines

69 (Polkinghorne et al. 2013; Rhodes et al. 2011). The arboreal folivorous koala is the sole extant

70 representative of Family Phascolarctidae, and an internationally recognized iconic species of high

71 conservation, sociocultural and economic value (Black et al. 2014; Hundloe and Hamilton 1997;

72 Price 2012). In recent decades widespread population declines have become apparent, particularly

73 in the northern parts of the koala's range (DERM 2009; McAlpine et al. 2015; Melzer et al. 2000b).

74 Koalas are now listed as 'Vulnerable’ since 2012 under Australian Federal Government legislation

75 (Environment Protection and Biodiversity Conservation Act 1999; EPBC Act), across two states

76 and one territory (Queensland, New South Wales and the Australian Capital Territory; McAlpine et $77 \quad$ al. 2015). 
79 Successfully mitigating disease and managing koala populations to prevent extirpation and reverse

80 declines is a current priority for koala conservation. However, the relative impact of chlamydiosis

81 among other threats (including habitat loss, fragmentation, car collisions and dog attacks) is poorly

82 understood, and decision makers have been unable to effectively guide management policy because

83 there is no consensus regarding the nature and extent of the impact of chlamydial infections on

84 koala populations (McAlpine et al. 2015; Rhodes et al. 2011). Inadequate funding and technological

85 constraints may have limited understanding. Indeed, many fundamental aspects of the disease

86 ecology remain understudied (McCallum et al., under revision). To date, no studies have

87 quantitatively examined the breadth and scope of research related to koala chlamydiosis, nor

88 systematically identified the current research gaps.

89

90 With the goal of assisting policy planning for funding future koala chlamydial research, we have

91 used the well-established systematic quantitative review technique (Petticrew 2001; Pickering and

92 Byrne 2014; Rochman et al. 2016) to examine the extent of the peer-reviewed literature on koala

93 chlamydiosis. Our review examined general characteristics of papers/chapters included (such as

94 journal category, authorship, funding and spatiotemporal scope), their major foci and study types,

95 the breadth of chlamydial species covered, as well as methodological design (including data types,

96 sampling and analysis details). In the following sections we critically analyse the results, consider

97 study caveats, and identify research gaps that should be addressed in future studies. 
101 We used systematic search and compilation methodology common to quantitative systematic

102 literature reviews to provide a comprehensive and reproducible overview of the current status of

103 literature on koala chlamydiosis (Pickering and Byrne 2014; Rochman et al. 2016). Our purpose

104 was to evaluate the breadth and scope of the peer-reviewed literature available since 1970

105 (encompassing the first paper reporting aetiological diagnosis of chlamydiosis in koalas), including

106 identifying the presence of research trends and weaknesses, in order to highlight gaps to inform

107 policy and funding for future research agendas.

109 We searched Web of Science: All Databases (WoS; Thomson Reuters) and Scopus (Elsevier)

110 literature databases using 'title' or 'title, abstract, keyword' searches respectively, with date range

111 from 1 January 1970 to 28 April 2016. We initially examined all English-language literature

112 pertaining to the host species of interest, using the phrase “(koala* OR Phascolarct*)" (808 articles

113 from WoS, 1151 articles from Scopus), then narrowed these lists by including pathogen or disease-

114 associated terms “(diseas* or chlam* or cystit* or "wet bottom" or "dirty tail" or conjunctiv* or

115 keratoconjunc*)” (103 articles from WoS, 350 articles from Scopus). We assessed each of these

116 entries individually based on title and abstract (and where necessary, a more detailed assessment of

117 the paper) for relevance and inclusion in our database. We then modified this combined list with (i)

118 the addition of any relevant literature cited by the existing articles that was not originally included

119 in the database results, and (ii) specific inclusion and exclusion criteria (see Table 1).

121 Table 1. List of criteria governing the inclusion or exclusion of literature for the systematic

122 database.

123 


\begin{tabular}{|c|c|c|}
\hline Criteria & Publication characteristics & Study focus characteristics \\
\hline Inclusion & $\begin{array}{l}\text { - } \quad \text { Peer-reviewed primary literature } \\
\text { - } \quad \text { Peer-reviewed ‘conference } \\
\text { proceedings' if full papers } \\
\text { - } \quad \text { Edited book sections/chapters }\end{array}$ & $\begin{array}{l}\text { There must be reference to bacteria } \\
\text { of the Family Chlamydiaceae, and } \\
\text { infection of koalas (Phascolarctos } \\
\text { cinereus) OR } \\
\text { - There must be reference to clinical } \\
\text { signs and/or pathology of the koala } \\
\text { consistent with chlamydiosis }\end{array}$ \\
\hline Exclusion & $\begin{array}{l}\text { - } \text { Non-English language publications } \\
\text { - Abstract-only } \\
\text { publications/proceedings or editorial } \\
\text { pieces } \\
\text { - Study/paper duplicates } \\
\text { - Grey literature (popular articles, } \\
\text { theses, governmental reports) } \\
\text { - Literature not published between the } \\
\text { year } 1970 \text { and } 28 \text { April } 2016 \\
\text { - Peer-reviewed review and } \\
\text { modelling papers if they do not } \\
\text { present new empirical data in a } \\
\text { robust form }\end{array}$ & $\begin{array}{l}\text { - The reference to koala chlamydial } \\
\text { infection or disease must not be 'in } \\
\text { passing' (brief mention in } \\
\text { introduction/discussion), and must } \\
\text { instead be a main focus of the } \\
\text { literature } \\
\text { - We excluded those studies that did } \\
\text { not provide empirical results with } \\
\text { either quantitative or qualitative data } \\
\text { of relevance to chlamydial infection } \\
\text { or disease in koalas } \\
\text { - We excluded studies of C. pecorum } \\
\text { or C. pneumoniae if empirical data } \\
\text { did not include isolates from koalas }\end{array}$ \\
\hline
\end{tabular}

127 In this study we define koala chlamydiosis as any infection (or co-infection) of the host species $P$.

128 cinereus with bacteria of Family Chlamydiaceae. Importantly, this definition includes infection

129 with both the main species of culturable Chlamydia bacteria that have been identified as infecting

130 koalas to date (Chlamydia pecorum and C. pneumoniae), despite their putatively differing host

131 pathogenicity, as well as phylogenetic and geographic origins (Polkinghorne et al. 2013). It also

132 includes the potential infection with a suite of as yet uncharacterized and potentially unculturable

133 chlamydial species or strains that have been detected via genetic sequencing methodologies (Burach

134 et al. 2014; Devereaux et al. 2003). We have been deliberately inclusive and have not distinguished

135 between infections causing subclinical and clinical disease because a thorough recognition of the

136 transmission and infection dynamics with various chlamydial species will likely be crucial for

137 understanding the expression of clinical disease in koalas, and subsequent population-level impacts. 
139 In addition, we recognize four broadly inclusive disease syndromes related to the clinical signs and 140 gross pathology of chlamydiosis in the koala, including ocular keratoconjunctivitis, urinary tract

141 disease, reproductive tract disease, and respiratory disease (including rhinitis-pneumonia complex)

142 as described by Vogelnest et al. (2008). Development and widespread implementation of

143 appropriate diagnostic tests for atypical intracellular pathogens such as those in Family

144 Chlamydiaceae was a gradual process (for example, see Hammerschlag 1996). This constitutes a

145 bottleneck in the study of atypical emerging infectious diseases and poses challenges for defining

146 sufficiently comprehensive inclusion criteria when specific diagnostic testing is not yet consistently

147 performed. We thus chose to include the many relevant studies with a major focus on investigating

148 clinical signs and pathology that were highly consistent with our chlamydiosis disease syndromes

149 (see above), despite the absence of a clear aetiological diagnosis or pathogen isolation and

150 characterization. Our broad definition allows us to capture the variety of studies that have been

151 performed on chlamydial infections in koalas.

152

153 We were particularly interested in peer-reviewed original empirical data papers describing in situ 154 population-level chlamydial infection or disease dynamics in koalas. In general, we excluded 155 population-level demographic studies that made no specific mention of chlamydial infection or 156 disease signs in the results. Despite this focus, we recognize that key understanding has been 157 developed through studies predominantly focused on the pathogen in terms of phylogeny and 158 characterization, so these were included. We excluded both general review and modelling papers 159 from the database to eliminate overlapping data reporting, and 'unpublished data' from which their 160 results sometimes derive. Data were extracted from all publications included in the systematic 161 database by a single investigator for consistency, and were cross-checked and evaluated by all co162 authors. 
164 For each paper that met our inclusion criteria we thoroughly reviewed their content over several 165 occasions and constructed a database, recording data under the following headings: (i) literature 166 details, (ii) study focus, (iii) chlamydial species, (iv) study type, (v) koala sampling details, (vi)

167 koala source location, (vii) diagnostic methods, (viii) data type, and (ix) funding details. We

168 summarized and analysed the resulting database to detect patterns and also examined trends within

169 the groups defined by major study focus where this was relevant. However, we did not explicitly

170 apply weighting criteria to the various studies due to the breadth of research covered and lack of an

171 appropriately broad and inclusive rating scheme.

172

173 Under the 'Study focus' heading, we assessed papers for their main focus of either 'Koala' or

174 'Pathogen', and after examining the complete database, categorized studies into the following sub-

175 foci that best described their major purpose or aim. Under the 'Koala’ category: (i) population-level,

176 (ii) pathology, (iii) immunology, (iv) pharmacology, (v) other pathogen, (vi) diagnosis, and (vii)

177 experimental infection; and under the 'Pathogen’ category: (i) in vitro response, and (ii) phylogeny.

178 Several of these categories had to be relatively broad to encompass the diversity in the koala

179 chlamydial literature, and it is important to note that papers often reported data that were somewhat 180 peripheral to their research question or main focus.

181

182 The 'Koala' focused category included studies where koalas were directly or indirectly sampled or 183 observed for signs of chlamydial infection, disease, or diagnostic samples. This category included 184 studies advancing the diagnosis of koala chlamydiosis (and frequently included prevalence 185 reporting), but excluded studies predominantly characterizing the pathogen. The 'Pathogen’ focused 186 category included papers where the samples being investigated involved koala-specific

187 Chlamydiaceae, but were mainly concerned with characterizing the pathogen, without consideration 
188 of responses of koalas from which the samples or isolates were derived. To avoid paper overlap

189 between categories, where a paper focused on more than one major aim or purpose, we selected the 190 category that fit most closely.

191 


\section{Overall summary, disciplinary scope, authorship and funding}

195 From our comprehensive systematic review of the koala research literature between 1970 and 2016

196 (constituting 808 articles identified via WoS versus 1151 identified via Scopus), we identified a total

197 of 117 original peer-reviewed research papers that met our inclusion criteria (Table 1) pertaining to

198 koala chlamydial infection and disease (see Appendix S1 for complete list of studies examined and

199 details recorded). These papers were published in a total of 36 different journals and two books

200 (Fig. 1i and ii). The two journals individually publishing the highest number of papers were the

201 Australian Veterinary Journal (20 published papers), and the Journal of Wildlife Diseases (11

202 published papers).

(i) Journals by category

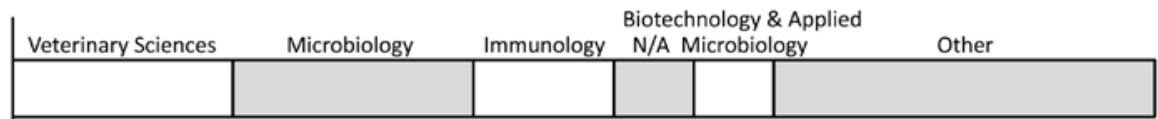

(ii) Papers by category

(iii) Publication decade

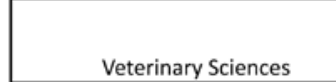

Biotechnology \& Applied

\begin{tabular}{l|l|ll} 
Veterinary Sciences & Microbiology Immunology N/A Microbiology Other
\end{tabular}

(ii) Papers by category
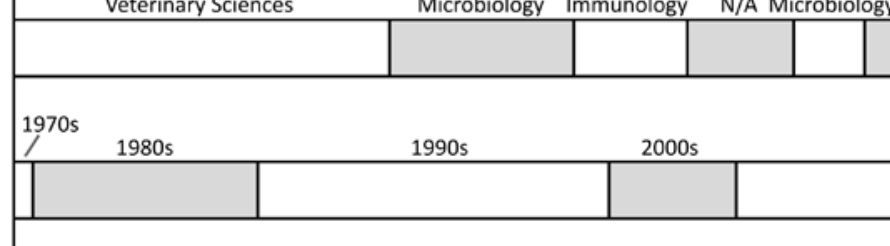

(iv) Chlamydial species

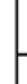

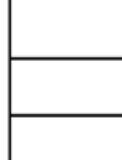

(v) Data type explored

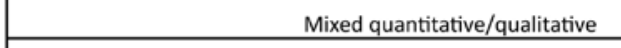

2000 s
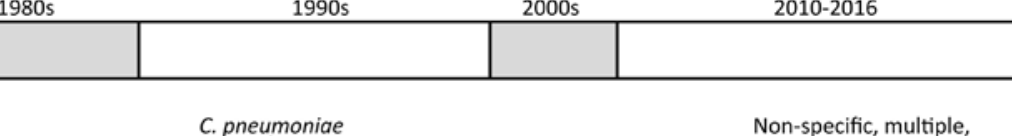

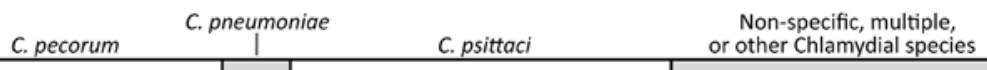

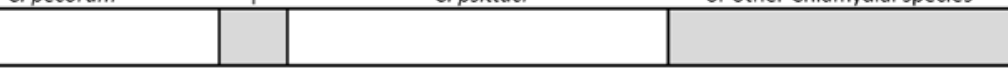

\section{.}

Mixed quantitative/qualitative Qualitative only

(vi) Major study foci

(vii) Minor study foci

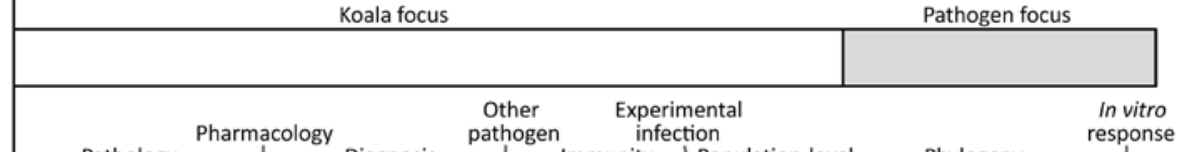

Pathology

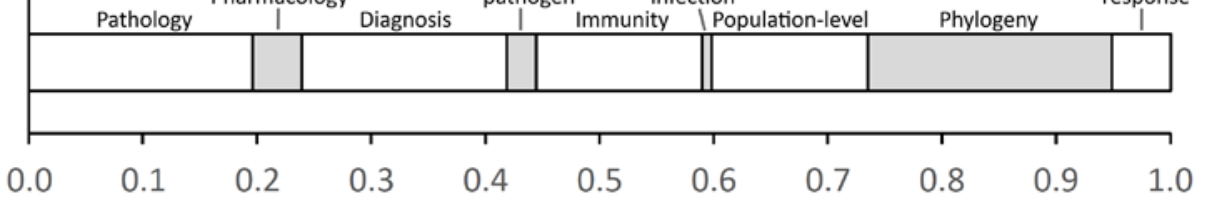

$\begin{array}{lllllllllll}0.0 & 0.1 & 0.2 & 0.3 & 0.4 & 0.5 & 0.6 & 0.7 & 0.8 & 0.9 & 1.0\end{array}$


205 Figure 1. Bar graph demonstrating the proportion of (i) journals grouped according to category, and

206 papers grouped by (ii) journal category, (iii) publication decade, (iv) chlamydial species examined,

207 (v) data type explored, (vi) major study foci, and (vii) minor study foci. Please note that Journal

208 categories used in (i) and (ii) above are not mutually exclusive (a journal may have more than one

209 category allocated according to Thomson-Reuters ${ }^{\mathrm{TM}}$ InCites ${ }^{\mathrm{TM}}$ Journal Citation Reports $\left.{ }^{\circledR}\right)$. Major

210 and minor study foci were mutually exclusive (only one major and one minor study focus allocated

211 per paper, please see supplementary information for more details).

212

213 These 117 papers represent the collective work of a total of 207 authors, who each contributed to

214 between 1 and 46 papers. The distribution of number of papers co-authored by specific researchers

215 was highly positively skewed (Fig. 2). The most highly represented authors ( $\geq 10$ papers) included

216 P. Timms (46 papers), A. Polkinghorne (23 papers), K. Beagley (17 papers), P. J. Canfield (15

217 papers), D. P. Higgins (13 papers), F. N. Carrick (12 papers), A. A. Girjes (12 papers), J. Hanger

218 (11 papers), and M. F. Lavin (11 papers). The profile of first-authorship varied from that of all co-

219 authors combined, with a range from between 1 and 10 papers first-authored by a single individual.

220 The authors with the highest first-author representation ( $\geq 4$ papers) included P. J. Canfield (10

221 papers), A. A. Girjes (7 papers), A. Kollipara (4 papers), and R. W. Martin (4 papers). The most

222 highly represented co-authors were not typically highly represented as first-authors: P. Timms

223 (first-author on 1 paper), A. Polkinghorne (0 papers), K. Beagley (0 papers), P. J. Canfield (10

224 papers), D. P. Higgins (3 papers), F. N. Carrick (0 papers), A. A. Girjes (7 papers), J. Hanger (1

225 paper) and M. F. Lavin (1 paper).

226 


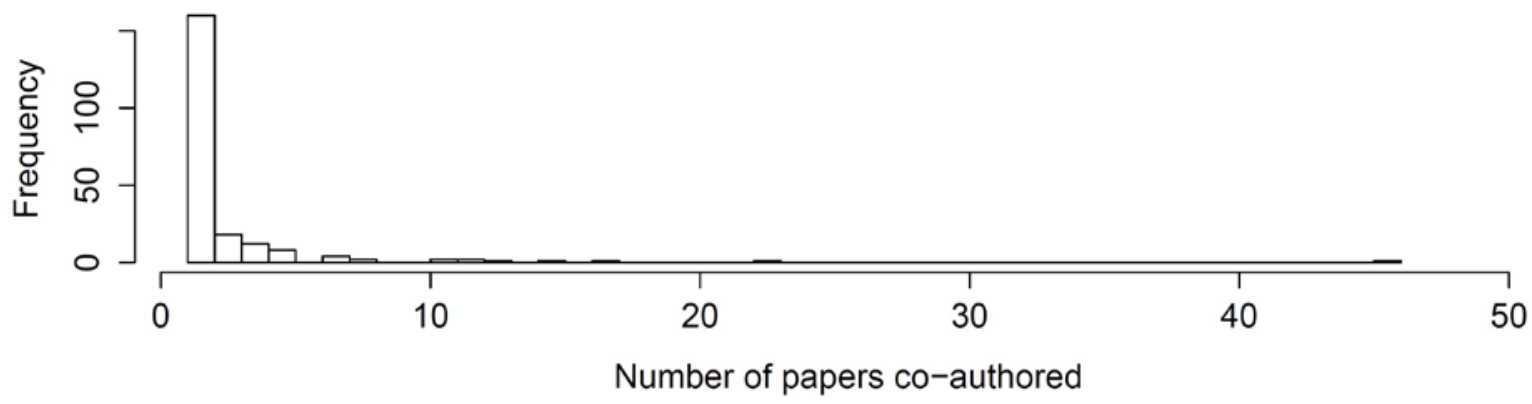

Figure 2. Histogram of the frequency of authors represented by the number of papers they co-

229 authored.

231 The majority (97) of studies specifically acknowledged funding agencies, organizations or schemes

232 for financial support, and the funders listed were diverse (and included a number of international

233 funders from USA, Japan, UK, Iraq, etc.). A total of 37 papers reported funding from the Australian

234 Research Council (ARC; with 20 specifically acknowledging ARC Linkage grants and 7

235 acknowledging ARC Discovery Projects). The next most prolific funders are listed in Table 2, with

236 the remaining funders attributed in four or fewer papers.

237

238 Table 2. Funding agencies or schemes most frequently attributed for financial support (those

239 attributed by five or more papers; funder attribution was not mutually exclusive).

\begin{tabular}{lc}
\hline Major funding agencies or schemes attributed & Frequency \\
\hline Australian Research Council (ARC) & 37 \\
Australian Koala Foundation (AKF) & 15 \\
Lone Pine Koala Sanctuary & 13 \\
National Parks and Wildlife Service (NPWS) & 11 \\
The University of Queensland Koala Fund (UQKF) & 9 \\
Queensland Government National and International Research & 7 \\
Alliances Program Scheme (NIRAP) & 7 \\
Sun Herald Koala Appeal Fund & 5 \\
Queensland Department of Environment and Heritage Protection & 5 \\
Koala Research Grant (QDEHP) & \\
\hline
\end{tabular}




\section{Spatiotemporal breadth and key koala source locations}

242 We purposely selected the temporal range of our review (1 January 1970 to 28 April 2016) to

243 include the earliest peer-reviewed publication that reported an aetiological diagnosis of chlamydial

244 infection in koalas (Cockram and Jackson 1974; published in the Australian Veterinary Journal).

245 Two papers were published in 1970s, 23 papers in 1980s, 36 papers in 1990s, only 13 papers were

246 published in 2000s, and 43 papers were published from 2010-2016 (Figs. 1iii and 2).

247

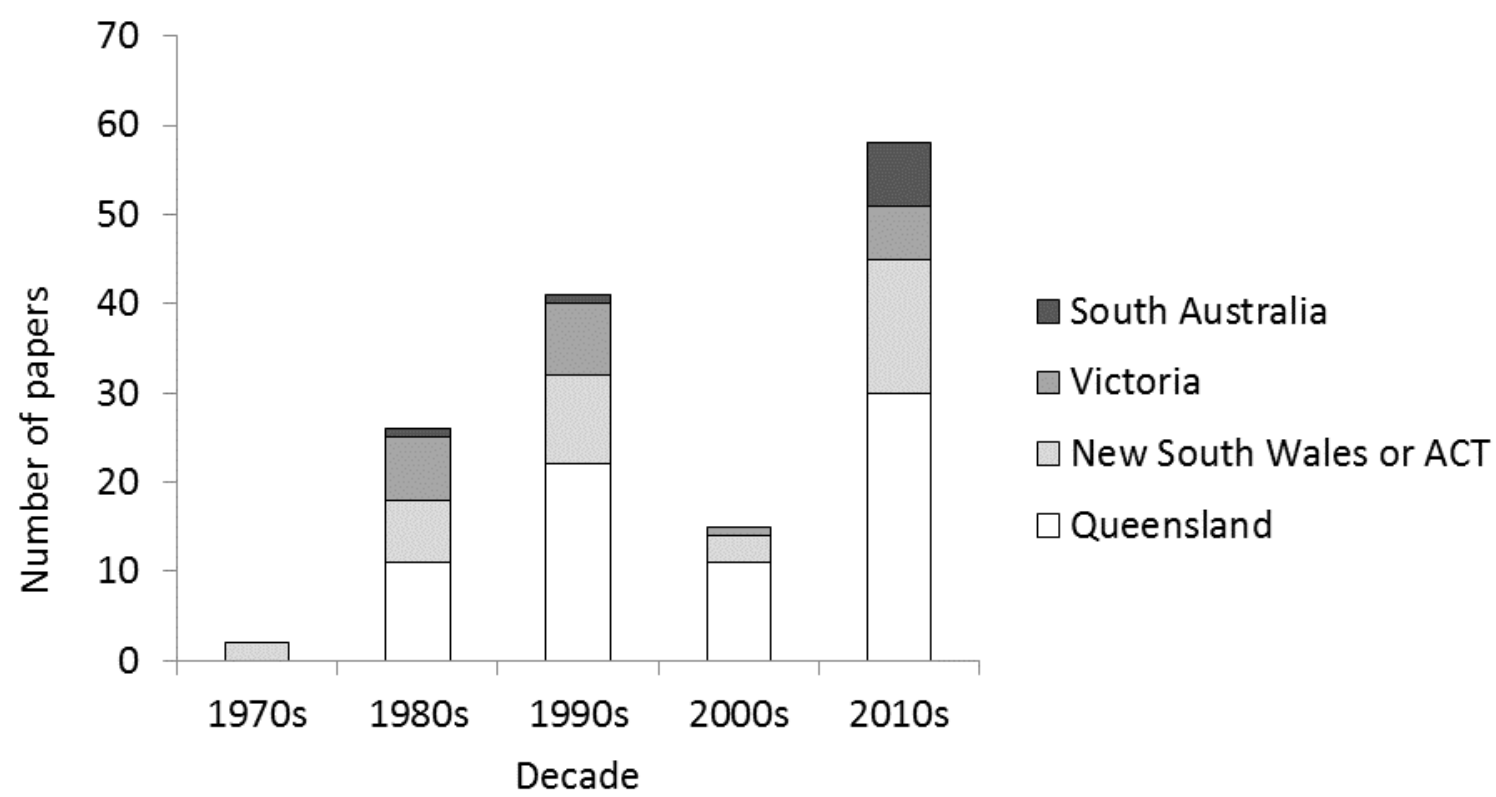

249 Figure 3. Number of papers primarily based on data from each state/territory by decade (not

250 mutually exclusive - some papers used koala or chlamydial isolate data collected from multiple

251 states).

252

253 Seventy-four studies contained koala samples, data or chlamydial isolates from Queensland (59 of

254 which were solely QLD data), 37 with data from New South Wales and/or the Australian Capital

255 Territory (21 of which were solely NSW/ACT data), 22 with data from Victoria (15 solely VIC), 
and nine studies with data from South Australia (only 2 solely SA) (Fig. 3). Four studies contained

257 insufficient detail on the koala Chlamydia isolates used to determine their geographic origin.

259 Several key study locations (captive sanctuary or hospital situations) were identified as sources for

260 koalas, samples, or chlamydial isolates in many studies (Fig. 4). These included most frequently

261 Lone Pine Koala Sanctuary, QLD (approximately 15 studies), Port Macquarie Koala Hospital,

262 NSW (15), Australia Zoo Wildlife Hospital, QLD (10), Queensland Parks and Wildlife Service

263 Koala Hospital Moggill, QLD (7), Featherdale Wildlife Park (4), Dreamworld, QLD (2) and

264 Currumbin Sanctuary, QLD (2) (several studies were unclear, and some included many sources).

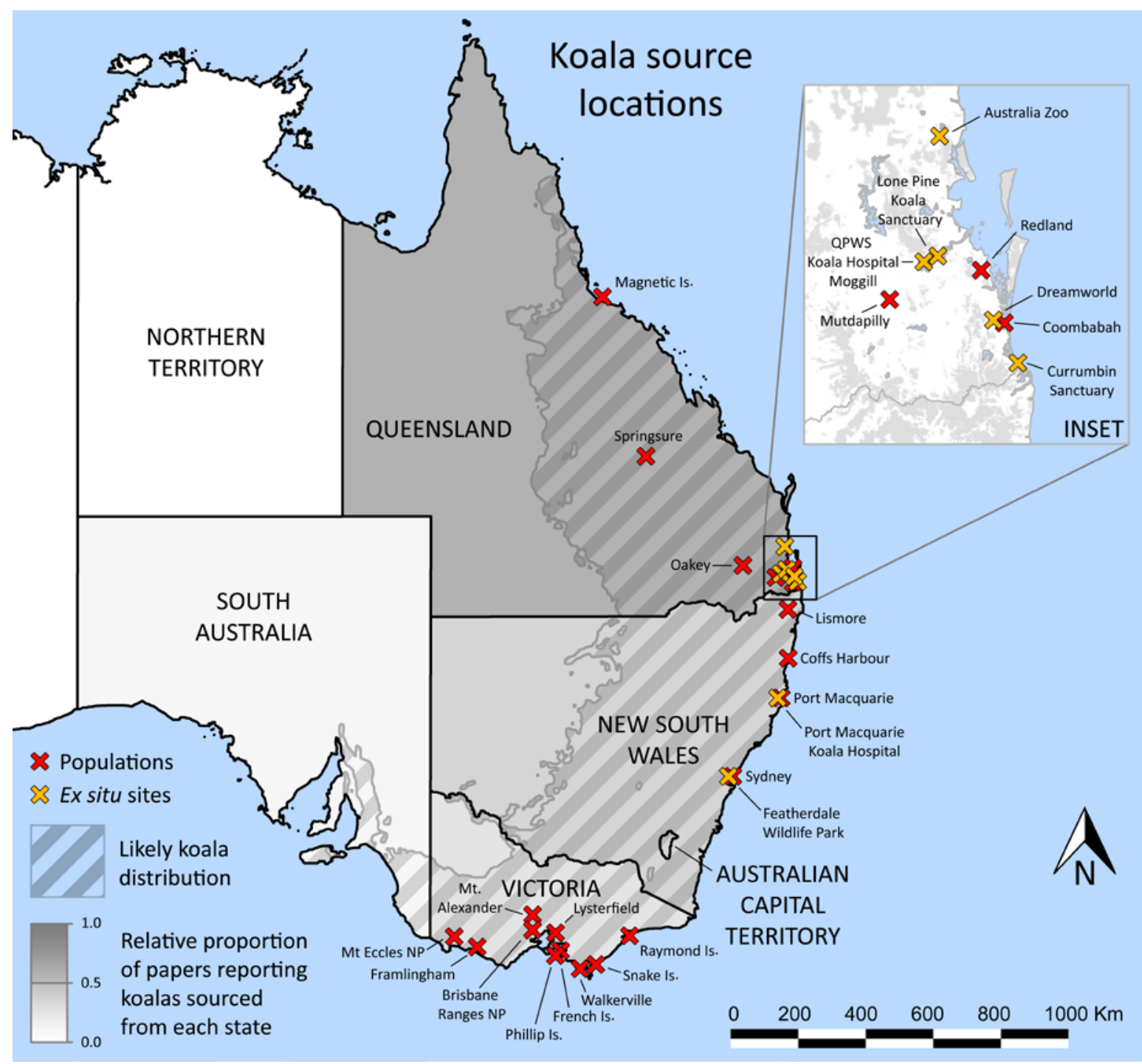


266 Figure 4. Map of the key source locations for sampled koalas in Australia (red crosses =

267 populations sampled in the 16 population-level studies; orange crosses = key captive or hospital ex

268 situ sites), plotted against the probable maximum extent of koala distribution (diagonal shading),

269 modified from Threatened Species Scientific Committee (2012). States are shaded according to the

270 relative proportion of the total papers (117) that reported on data from koalas or chlamydial isolates

271 sourced from each state (see text for further details). The inset shows the south-east Queensland

272 region surrounding Brisbane in greater detail. QPWS = Queensland Parks and Wildlife Service.

273

274 Koala subjects - context, sampling size and sampling robustness

275 The distribution of the number of koalas examined per paper was strongly positively skewed (Fig.

276 5). Data from a total of up to 11,027 koalas were reported in the studies identified (assuming no

277 overlap between individual koalas and koala samples), with an average of 94 koalas sampled or

278 examined per paper. However, the majority of studies examined data from 27 (median) or fewer

279 koalas (18 studies examined chlamydial isolates, samples, or data from three or fewer koalas), with

280 only four studies examining more than 500 koalas. The maximum number of koalas reported in a

281 single paper was 2,674 in a 30 year retrospective analysis of hospital admission data (Griffith et al.

282 2013). Examining only the 16 population-level studies, this average was 177 (median = 99, total

283 across these studies = 2,837). Overall, 41 papers involved captive koalas, while 87 involved free-

284 ranging koalas (25 studies involved both), the majority of which were brought in opportunistically

285 through passive surveillance for care at koala hospitals by members of the community rather than

286 being sampled in the field. Fourteen studies were unclear on whether the koalas from which isolates

287 were used were free-ranging or captive. 


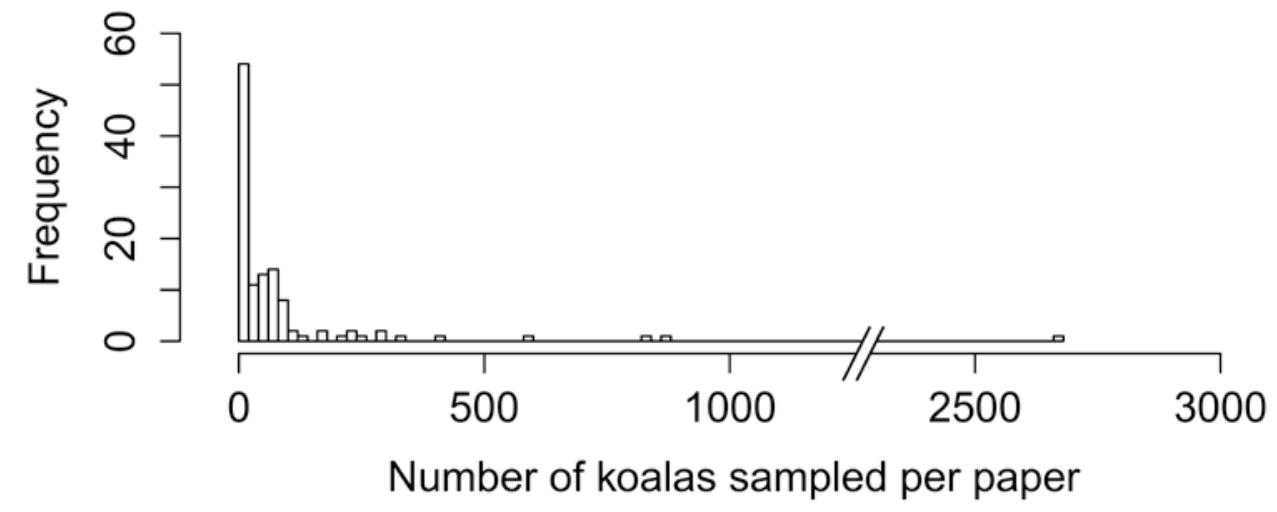

289 Figure 5. Frequency histogram of the frequency of papers associated with the number of koalas 290 sampled per paper (bin width $=20$ koalas).

We assessed 107 (91\%) papers as presenting data from opportunistically sampled koalas. This category included studies involving koalas admitted to hospitals, captive koala populations, archived sample specimens, individual chlamydial isolates, or poorly reported field-collected samples. While 33 studies mentioned field-sampled animals, only 10 of these reported robust field sampling methodologies, design and epidemiological analysis.

\section{Diagnostic testing performed and chlamydial species examined}

While most papers (98) included some form of Chlamydia aetiological diagnostic testing (including

300 a suite of tests from the earliest yolk sac isolation tests, to the current best practice methods of

301 quantitative polymerase chain reaction (qPCR) for chlamydial antigens, and enzyme-linked

302 immunosorbent assay (ELISA) for serum antibodies; Carey et al. 2010; Markey et al. 2007), 15

303 papers only contained diagnosis of chlamydial infection or disease on the basis of consistent clinical

304 signs, pathological evidence (gross or histological), or radiographic/ultrasonographic evidence for

305 signs of reproductive tract disease. These papers were included because appropriate aetiological

306 diagnostic tests were not routinely available in the early years of chlamydial investigations. A

307 diverse range of methodologies was reported for diagnosis, comparison or investigation (Table 3). 
308 Examination of clinical signs of disease (either physically captured/handled animals, or distance

309 observations in situ) was the most commonly reported diagnostic method.

310

311 Table 3. Diagnostic methods and other specific laboratory techniques, and the total number of times

312 they were reported in the full set of papers (not mutually exclusive).

313

\begin{tabular}{|c|c|c|c|}
\hline General category & Specific technique & Frequency & Total \\
\hline \multirow{8}{*}{$\begin{array}{l}\text { Routine veterinary } \\
\text { diagnostics }\end{array}$} & Clinical signs & 36 & \\
\hline & Gross pathology & 23 & \\
\hline & Haematology & 9 & \\
\hline & Ultrasonography & 5 & \\
\hline & Radiography & 4 & \\
\hline & Blood biochemistry & 4 & \\
\hline & Ectoparasite examination & 1 & \\
\hline & Urinalysis & 1 & 83 \\
\hline \multirow{5}{*}{$\begin{array}{l}\text { Culture or isolation } \\
\text { methods }\end{array}$} & Cell culture & 21 & \\
\hline & Aerobic culture & 7 & \\
\hline & Chick embryo yolk sac isolation & 4 & \\
\hline & Anaerobic culture & 3 & \\
\hline & Fungal culture & 1 & 36 \\
\hline \multirow{5}{*}{ Microscopy } & Histopathology & 25 & \\
\hline & Immunohistochemistry & 8 & \\
\hline & Cytology on smears or wet mounts & 6 & \\
\hline & Electron microscopy & 4 & \\
\hline & Direct immunofluorescence & 4 & 47 \\
\hline \multirow{10}{*}{$\begin{array}{l}\text { PCR and genetic } \\
\text { methods }\end{array}$} & Polymerase chain reaction (PCR) & 18 & \\
\hline & Quantitative PCR (qPCR) & 18 & \\
\hline & Gene, genome or DNA sequencing & 18 & \\
\hline & Gene and DNA probe or cross-hybridization & 11 & \\
\hline & Restriction enzyme analysis & 4 & \\
\hline & High resolution melt curve analysis & 2 & \\
\hline & Culture-independent DNA capture & 1 & \\
\hline & Microarray & 1 & \\
\hline & PCR amplicon sequencing & 1 & \\
\hline & Chlamydia multi-locus sequence analysis & 1 & 75 \\
\hline \multirow{3}{*}{$\begin{array}{l}\text { Serology and } \\
\text { specific tests }\end{array}$} & Complement fixation test (CFT) & 22 & \\
\hline & Enzyme-linked immunosorbent assay (ELISA) & 21 & \\
\hline & Lymphocyte stimulation assay & 9 & \\
\hline
\end{tabular}


Western blotting 8

DNA or immuno dot or slot blot 7

In vitro Chlamydia neutralization $\quad 6$

Pepscan methodology 2

CFT with whole blood sampling paper $\quad 1$

Flow cytometry 1

Other/non-specific $\quad 7$

315 Four papers did not contain any individual-level aetiological, clinical, pathological, radiographic or

316 ultrasonographic diagnosis of Chlamydia (Hernandez-Sanchez et al. 2015; Martin and Handasyde

317 1990; Martin 1985b; McLean and Handasyde 2006). These papers mostly reported chlamydial

318 infection or disease at the population level, including either unpublished data summaries, or

319 unsubstantiated claims of particular populations being 'Chlamydia-free'. Hernandez-Sanchez et al.

320 (2015) reported a retrospective vaccine survival study with no chlamydial diagnostic testing

321 performed (although the vaccine was produced based on sequenced rMOMP of C. pecorum).

323 We grouped papers by the major chlamydial species studied (Fig. 1iv). Chlamydia pecorum was the

324 major focus of 35 papers, C. pneumoniae in 7 papers, C. psittaci in 39 papers, and 'non-specific,

325 multiple or other chlamydial species' in 36 papers. In addition to non-culturable Chlamydiaceae,

326 this latter category included all studies where chlamydiosis was diagnosed on the basis of clinical

327 signs of disease or histopathology of infection, in the absence of any specific aetiological diagnostic

328 test for chlamydial species. The C. psittaci category contained all early studies, prior to the separate

329 identification of the two main infecting chlamydial species (C. pecorum and C. pneumoniae;

330 Glassick et al. 1996). Due to earlier reporting, infections were assumed to be caused by a single $C$.

331 psittaci pathogen species. Here we have included any reports of Chlamydophila species together

332 with the respective Chlamydia species, as the change in taxonomic naming that occurred in the

333 early 2000s was not widely accepted and has since been abandoned (Devereaux et al. 2003;

334 Polkinghorne et al. 2013). 


\section{Study and data types}

337 We examined papers for the type of study performed, including field, hospital/captive, experimental 338 laboratory, and computer analysis components (not mutually exclusive) (Fig. 1v). The majority of 339 studies involved laboratory experimental analyses in vitro (either performed on samples or isolates;

340 74), and approximately half of all studies utilized hospital or captive population koala samples (61).

341 There was only one experimental infection study undertaken in a captive environment (Brown

342 1986). Ten studies involved the use of some form of developmental chlamydial vaccine, and hence

343 additionally constituted experimental manipulation of animals, with or without natural infection in

344 the wild. There were five studies involving treatment trials, however the majority of

345 hospital/captive population studies involved necropsy case studies (28) or sample collection (37).

346 Less than a third of studies involved a field component (33), and most of these reported sample 347 collection (27) with minimal corresponding ecological data or collection methodology (such as 348 mark-recapture, which was reported in 8 papers; or radio-tracking, reported in 5). There were five 349 studies that involved some form of field koala translocation (Khan et al. 2016; Lee et al. 1990;

350 McColl et al. 1984; Obendorf and Handasyde 1990; Waugh et al. 2016). A computer-based

351 bioinformatics genetic analysis component was performed in 24 studies (phylogenetic pathogen-

352 focused studies), while there were four studies based on retrospective computer records. 353

354 The majority of studies used mixed quantitative/qualitative results reporting (95 mixed, 22

355 qualitative only), although most of these only presented summary (descriptive) statistics and didn’t

356 perform hypothesis testing statistics or model evaluations. We found 39 studies (excluding

357 phylogenetic analyses, sensitivity/specificity testing, and qPCR results reporting) that performed

358 and reported on specific statistical testing procedures. 
361 While the majority of included studies (85) focused predominantly on koalas (Fig. 1vi) (compared

362 with only 32 focused on specific koala chlamydial pathogens), most of these involved outcomes at

363 the individual-host level (Figs. 1vii and 5): pathology (23 papers), diagnosis (21), immunity (17),

364 pharmacology (5), other pathogen (3), and experimental infection (1). Only 16 studies (14\% of all 365 studies) focused on population-level disease dynamics or impacts. Of papers/chapters that focused 366 on the pathogens, most investigated phylogeny (25) compared with only six that characterized in 367 vitro responses.

368

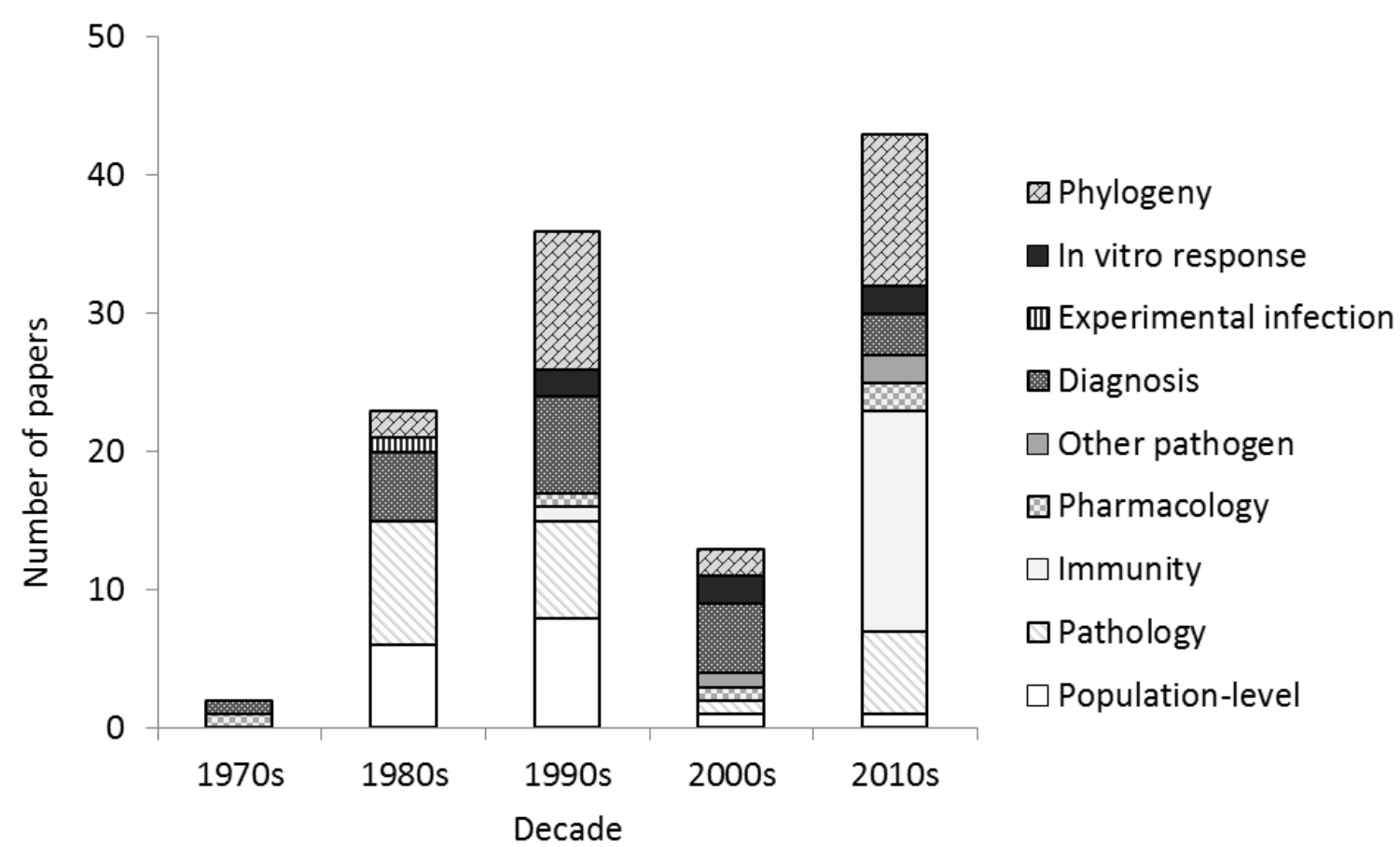

369

370 Figure 6. Number of papers published in each of the minor study foci by decade (minor study foci

371 categories are mutually exclusive - a paper is only allocated to one minor study focus).

372

373 We classified papers into the 'Pathology' category (23 papers) if they investigated the impact of

374 infection and disease on the individual host using predominantly veterinary diagnostic pathology 
375 techniques (reported as case studies or series). These were proportionally over-represented in the

376 early years of koala chlamydial investigations and mildly under-represented in later years (9 papers

377 in 1980s, 7 in 1990s, 1 in 2000s and 6 in 2010s) (Fig. 6). Most pathology studies originated from

378 NSW (14), with fewer from the other states (5 from QLD, 4 from VIC, 2 from SA).

379

380 Other studies that aimed to develop and evaluate methods of diagnosis for koala chlamydial

381 infection/disease were grouped into the 'Diagnosis' category (21 papers). The frequency of

382 publication of these studies peaked in the 1990s (8 papers) around the time of reclassification of

383 koala chlamydial species from C. psittaci firstly to C. psittaci type I and II, then to C. pneumoniae

384 and C. pecorum respectively (Girjes et al. 1994; Girjes et al. 1988; Glassick et al. 1996), with fewer

385 papers focused solely on diagnostic methods since the introduction of PCR techniques. Most of the

386 koalas sampled for these studies were obtained opportunistically through wildlife hospitals, zoos

387 and captive koala populations from QLD (15 papers), with only four studies from NSW and one

388 from VIC.

389

390 Studies examining mechanisms of immunity or immune-based mitigation techniques (vaccination)

391 fell within the 'Immunity’ category (17 papers). Since 2010 these studies were mostly performed in

392 the northern parts of the koala’s range (15 papers with koalas sourced from QLD, 2 papers with

393 koalas from NSW).

394

395 We only identified 16 'Population-level' studies that focused on population-level data to assess the

396 extent and impact of chlamydiosis on free-ranging koala populations and other epidemiologic

397 parameters. These were proportionally over-represented in the early years (4 studies published in

398 1980s, 8 papers in 1990s, one paper in 2006 and one paper published in 2015). The geographic

399 spread of the studied populations was also limited, with most studies performed on Victorian 
400 populations (8), followed by seven studies on QLD populations, and one from NSW. The

401 geographic locations of these studied populations are identified in Fig. 1.

402

403 A small number of studies were identified that examined pharmacology related to treatments for

404 koala chlamydial infections (5), or identified correlations between chlamydiosis and other

405 pathogens (including trypanosomes, koala retrovirus or herpesvirus; McInnes et al. 2011; Stalder et

406 al. 2015; Tarlinton et al. 2005). Brown and Grice (1986) presented the only data involving

407 experimental infection in a controlled environment, and no papers presented empirical data on

408 natural routes of infection transmission either ex situ or in the field.

409

410 From the studies focused predominantly on the chlamydial pathogens, 25 involved phylogenetic

411 characterization, most commonly published in the last decade (11 papers from 2010s), and almost

412 invariably including koala chlamydial samples or isolates from QLD (18 papers), although

413 geographic sources were diverse in many. A further six studies focused on characterizing koala

414 chlamydial pathogens in vitro, including morphology, growth, environmental factors, and survival

415 responses to antifungal treatments.

416 
DISCUSSION

419 The objective of this quantitative systematic review was to consider the breadth of research 420 performed on koala chlamydiosis within the context of recent koala population declines. We thus

421 mapped the development of research in the field with regards to journal category, authorship,

422 funding, spatiotemporal scope, study foci and type, chlamydial species examined, methodological

423 design and overall findings. Here we critically evaluate trends in the existing literature and identify

424 directions for future research in order to assist with policy planning and to guide research funding.

\section{Limited population-level disease data for koala chlamydiosis}

427 In a research field motivated largely by sociopolitical concerns for both the conservation of an 428 iconic species and the welfare of its individuals (McAlpine et al. 2015), it was striking to find 429 comparatively few studies reporting empirical data that were appropriate for evaluating population430 level impacts of chlamydiosis in the context of population declines. Of the 117 papers included in 431 our review database, only 16 focused on epidemiological population-level data, aside from 432 proportional infection measures from opportunistically sampled individuals. While several other 433 papers reported a field component to their study, these were mostly based on samples collected 434 from field-caught koalas without any indication of sampling methodology or other demographic 435 parameters.

437 Population-level disease studies were spatiotemporally restricted

438 Of particular concern, only three population-level disease studies were identified as published in the 439 last two decades (Jackson et al. 1999; McLean and Handasyde 2006; Patterson et al. 2015).

440 However, prior to the mid-1990s, diagnostic techniques for differentiating chlamydial species and 441 strains were not available (qPCR; Markey et al. 2007), and further, the two main species of 
Chlamydia infecting koalas had not yet been recognized as separate entities (Chlamydia pecorum

443 and C. pneumoniae; Girjes et al. 1994; Glassick et al. 1996). Additionally, over half of these studies

444 were performed on Victorian populations (including four island sites) that are arguably of lower

445 concern with regard to disease linked declines (Melzer et al. 2000a), with even fewer studies

446 performed in NSW. Consequently, our appreciation of in situ infection dynamics for the different

447 chlamydial species across the koala's distributional range is severely limited. Unfortunately,

448 ecological studies that examine population demography independent of reporting infection and

449 disease data are unable to provide the necessary detail.

451 Population-level disease studies were methodologically limited

452 Observational cross-sectional and comparative population-level studies were the most common, but 453 unfortunately these methodologies are readily confounded as per the ecological fallacy when used

454 to investigate disease (Clancy et al. 2003; Wobeser 2007). Only eight papers reported some form of 455 mark-recapture/re-sight methodology, and three population-level studies reported radio-tracking

456 individuals. The longest study was performed by Gordon et al. (1990) with annual surveys over 15

457 years. Other cohort studies ranged from as little as three weeks of observation data (Hirst et al.

458 1992), to approximately three years (Lee et al. 1990; Martin 1985a, b), with one other study

459 comprising two point-surveys of marked animals five years apart (Mitchell et al. 1988). Most

460 population-level studies reported measures of fecundity in association with disease (for example

461 McColl et al. 1984; Patterson et al. 2015), however, there was little focus on other demographic

462 parameters, such as estimates of population-level survival probabilities and population growth rates

463 in the context of disease (for example Gordon et al. 1990; Martin and Handasyde 1990). Most

464 studies were also deficient in description of koala sampling methods, lacking details of study site,

465 spatio-temporal sampling regimen and protocols, summaries of individual animal data or sub-

466 population structure, and justifications regarding potential bias and confounding (for an example of 
467 a study that does provide these details, see White and Kunst 1990). Without further detail we were

468 unable to confirm robustness to sampling bias and had to assume that their sampling was

469 opportunistic.

471 Future directions for population-level disease studies on koala chlamydiosis

472 Appropriate population-level data are crucial for wildlife disease studies for two reasons: (i) they

473 provide ecological validity by elucidating disease mechanisms in the stochastic context, complexity

474 and correct scale of the real-life system, and (ii) they generate supporting ecological data

475 (demography and population-structure) that are essential for identifying causes of heterogeneities of

476 infection and disease at the population scale. The relative lack of population-level koala

477 chlamydiosis studies identified in this review may be due to the following reasons. First, field

478 studies are expensive to perform, particularly at a spatiotemporal scale appropriate for the species'

479 autecology (relatively large distributional ranges and long generational intervals; Schneider 2001),

480 and funding for wildlife disease studies is typically scarce (Grogan et al. 2014). Second, field-based

481 disease studies are logistically complex requiring transdisciplinary expertise and resources in order

482 to ensure representational and ethically appropriate disease sampling (Wobeser 2007).

483

484 Strengthening the diversity and currency of population-level disease studies on koala chlamydiosis

485 should be a priority for future research. Greater emphasis should be placed on manipulative

486 population-level studies and longitudinal studies, using methodologies such as mark-recapture and

487 tracking in order to better elucidate infection mechanisms and parameters, as well as improving data

488 on basic koala demographic rates in association with disease (particularly robust measures of

489 infection-state associated survival, recruitment and population growth; Cormack 1964; Pradel

490 1996). Population-level manipulative experiments, in which pathogens or parasites are managed in

491 some populations with other populations used as controls, are rarely undertaken, largely due to 
expense, logistical challenges (for example, see Marcus et al. 2015), and the difficulty of identifying

493 Chlamydia-free populations. They are, however, essential to determine whether compensatory

494 effects to parasite-induced impacts on fecundity or survival exist. One of the few examples in the

495 literature is the classic study by Hudson et al. (1998), which demonstrated that population cycles in

496 red grouse are driven by nematodes by treating some populations with anthelmintics whilst keeping

497 others as controls.

499 Multi-state mark-recapture studies incorporating sampling for qPCR and serology can provide

500 probability estimates for transition to and from infection and disease states (Cooch et al. 2012;

501 Lebreton and Pradel 2002). Such approaches have proved critical in understanding disease threats in

502 wild populations (see, for example Faustino et al. 2004; Lachish et al. 2010; Murray et al. 2009).

503 Mark-recapture data should also be collected in association with information on individual

504 covariates (particularly infection and disease states, age and gender; Bonner et al. 2010). Disease-

505 state occupancy surveys and other spatial techniques may also assist in better understanding the role

506 of source-sink dynamics on the transmission of infection and manifestation of disease across

507 populations in a range of environments and under a range of threatening processes (Adams et al.

508 2010; Cashins et al. 2015; Mackenzie et al. 2009). Given the generally poor long-term efficacy of

509 chlamydial vaccines in multiple species (Entrican et al. 2012; Rey-Ladino et al. 2014), research into

510 alternative field-based management options may also be beneficial in addition to the current koala

511 vaccine studies (Khan et al. 2016; Waugh et al. 2016). Another area that warrants further

512 investigation is the putative association of chlamydiosis with other diseases (such as trypanosomes,

513 herpesvirus and Koala Retrovirus; McInnes et al. 2011; Stalder et al. 2015; Tarlinton et al. 2005). In

514 particular, longitudinal studies may help tease apart the direction of causality and the nature of

515 association.

516 


\section{Koala chlamydiosis research was otherwise diverse and reflects development of the field}

518 Aside from the limited population-level disease studies, the published literature on koala

519 chlamydiosis was diverse. Trends within the work appear to be a likely consequence of either the

520 existing expertise within the field, or the changing needs in the development of the field with

521 improving technologies and diagnostic capabilities. For example, there was an early focus on

522 pathology and diagnosis, and a late focus on immunity and pathogen phylogeny. The number of

523 papers published in the field has generally increased over the last four decades as expected with an

524 emerging field, but there was an unexpected dip after the turn of the century (Fig. 3). This temporal

525 shift may have been associated with a change in funding availability, commencement or progress of

526 long-term studies, or a change in the perceived importance of koala chlamydiosis at that time.

527 Spatially, the source of data was biased towards the northern parts of the koala's range, in accord

528 with indicators of population decline. Several study locations, both private and government funded

529 hospitals and sanctuaries, featured as major sources for koalas and samples. Pathology studies were

530 highly represented by koalas sampled from NSW, and diagnostic and immunity studies were

531 predominantly from QLD, possibly due to the range of hospital and captive sites providing access to

532 koalas (Fig. 4), and the perceived need for mitigation measures in declining populations. While

533 widely distributed geographic sampling was reported in a range of papers, these were

534 predominantly pathogen phylogenetic studies that deliberately sought a geographically diverse

535 range of samples, in a non-systematic fashion for comparative purposes.

537 A broadly transdisciplinary focus for the included papers and journals was identified, and this is

538 common to the study of wildlife diseases due to the overlap of ecology, veterinary and conservation

539 biology disciplines. Many authors were represented throughout the published literature, but some

540 individuals had a disproportionate role in the field likely due to their involvement in driving

541 particular avenues of research expertise in their respective teams. Funding was diverse, and 
542 interestingly, private organisations and overseas funders were attributed more often than we

543 expected. This might suggest that the iconic status of koalas attracts private funding, but possibly

544 also contributes to bias in the development and direction of the research field.

545

546 The relative lack of hypothesis-testing manipulative experiments with live koalas (including

547 treatment trials, translocation studies, vaccine reintroduction studies and infection experiments;

548 Brown and Grice 1986; Govendir et al. 2011; Lee et al. 1990; Markey et al. 2007; Waugh et al.

549 2016) was surprising, but a likely consequence of the ethical and logistical challenges of working

550 with an iconic arboreal specialist species, and a reflection of top-down priorities for funding.

551 Otherwise, the diversity of study and data types was largely expected although we were surprised

552 by the predominance of opportunistically sampled koalas (particularly the large number of studies

553 utilizing koalas and koala samples from hospitals), and relatively small sample sizes (median of 27

554 koalas per study). We also identified a general absence of substantiation for claims that populations

555 are 'Chlamydia-free'. Sampling strategies have been developed in other disease systems for proving

556 absence of infection and disease, and these should be adapted where possible (Caporale et al. 2012;

$557 \quad$ Skerratt et al. 2008).

558

559 Studies investigating diagnostic techniques made up a large proportion of published papers,

560 particularly in 1980s-2000s (Fig. 6). This was likely due to the inherent difficulties in accurately

561 diagnosing an intracellular bacterial pathogen, the gradual development of technologies, and two

562 key events: (i) the identification of two main chlamydial species infecting koalas (C. pecorum and

563 C. pneumoniae), as opposed to the originally identified C. psittaci (Cockram and Jackson 1974;

564 Glassick et al. 1996), and (ii) taxonomic renaming of the main genus of interest from Chlamydia to

565 Chlamydophila, and then back again (Bavoil et al. 2013; Devereaux et al. 2003; Polkinghorne et al.

566 2013). After the differentiation of chlamydial species, the majority of studies (35) focused 
exclusively on C. pecorum, likely due to its demonstrated higher pathogenicity (Jackson et al. 1999), compared with only seven exclusively focused on C. pneumoniae (usually in comparison with human isolates; Mitchell et al. 2010; Myers et al. 2009).

\section{Conclusions and future directions}

572 In this quantitative systematic review we critically analysed trends in the published peer-reviewed 573 English-language literature pertaining to koala chlamydiosis from 1970 to 2016. In particular, we

574 identified a striking lack of recent population-level disease studies, which makes evaluating the role 575 of chlamydiosis in recent population declines difficult. Hypothesis-testing manipulative

576 experiments for elucidating disease dynamics were also comparatively poorly represented. We

577 recommend a greater emphasis in future studies on elucidating population-level disease

578 mechanisms and parameter estimation, particularly through the use of manipulative and longitudinal

579 studies at appropriate spatiotemporal scales (Schneider 2001) and sample sizes (Griffith et al. 2013)

580 relative to the ecology of the koala.

581

582

\section{ACKNOWLEDGMENTS}

583 The authors thank Alison Peel for helpful comments on the manuscript. The project was funded by

584 “Investing to protect our koalas” - Koala Research Grant Program 2012, Department of

585 Environment and Heritage Protection, Queensland Government, Australia.

\section{REFERENCES}

2012. Phascolarctos cinereus (Koala) Listing Advice. Threatened Species Scientific Committee. Australian Government Department of Sustainability, Water, Population and Communities, Canberra, ACT.

Adams, M.J., Chelgren, N.D., Reinitz, D., Cole, R.A., Rachowicz, L.J., Galvan, S., McCreary, B., Pearl, C.A., Bailey, L.L., Bettaso, J., Bull, E.L., Leu, M., 2010. Using occupancy models to understand the distribution of an amphibian pathogen, Batrachochytrium dendrobatidis. Ecological Applications 20, 289-302.

Bavoil, P., Kaltenboeck, B., Greub, G., 2013. In Chlamydia veritas. Pathogens and Disease 67, 89-90. 
Black, K.H., Price, G.J., Archer, M., Hand, S.J., 2014. Bearing up well? Understanding the past, present and future of Australia's koalas. Gondwana Research 25, 1186-1201.

Bonner, S.J., Morgan, B.J.T., King, R., 2010. Continuous covariates in mark-recapture-recovery analysis: a comparison of methods. Biometrics 66, 1256-1265.

Brown, A.S., Grice, R.G., 1986. Experimental transmission of Chlamydia psittaci in the koala, In Chlamydial infections: Proceedings of the sixth international symposium on human chlamydial infections. eds D. Oriel, G. Ridgway, J. Schachter, D. Taylor-Robinson, M. Ward, pp. 349-352. Cambridge University Press, Sanderstead, Surrey.

Burach, F., Pospischil, A., Hanger, J., Loader, J., Pillonel, T., Greub, G., Borel, N., 2014. Chlamydiaceae and Chlamydia-like organisms in the koala (Phascolarctos cinereus)-Organ distribution and histopathological findings. Veterinary Microbiology 172, 230-240.

Caporale, V., Giovannini, A., Zepeda, C., 2012. Surveillance strategies for foot and mouth disease to prove absence of disease and absence of viral circulation. Revue Scientifique Et Technique-Office International Des Epizooties 31, 747-759.

Carey, A.J., Timms, P., Rawlinson, G., Brumm, J., Nilsson, K., Harris, J.M., Beagley, K.W., 2010. A MultiSubunit Chlamydial Vaccine Induces Antibody and Cell-Mediated Immunity in Immunized Koalas (Phascolarctos cinereus): Comparison of Three Different Adjuvants. American Journal of Reproductive Immunology 63, 161-172.

Cashins, S.D., Philips, A., Skerratt, L.F., 2015. Using site-occupancy models to prepare for the spread of chytridiomyosis and identify factors affecting detectability of a cryptic susceptible species, the Tasmanian tree frog. Wildlife Research 42, 405-413.

Clancy, K.J., Berger, P.D., Magliozzi, T.L., 2003. The ecological fallacy: Some fundamental research misconceptions corrected. Journal of Advertising Research 43, 370-380.

Cockram, F.A., Jackson, A.R.B., 1974. Isolation of a chlamydia from cases of keratoconjunctivitis in koalas. Australian Veterinary Journal 50, 82-83.

Cooch, E.G., Conn, P.B., Ellner, S.P., Dobson, A.P., Pollock, K.H., 2012. Disease dynamics in wild populations: modeling and estimation: a review. J Ornithol 152 (Suppl 2), S485-S509.

Cormack, R.M., 1964. Estimates of survival from the sighting of marked animals. Biometrics 51, 429-438.

Daszak, P., 2000. Emerging infectious diseases of wildlife - threats to biodiversity and human health. Science 287, 443.

DERM, 2009. Decline of the Koala Coast Koala Population: Population Status in 2008, Department of Environment and Resource Management. Queensland Government, Brisbane, Australia.

Devereaux, L.N., Polkinghorne, A., Meijer, A., Timms, P., 2003. Molecular evidence for novel Chlamydial infections in the koala (Phascolarctos cinereus). Systematic and Applied Microbiology 26, 245-253.

Entrican, G., Wheelhouse, N., Wattegedera, S.R., Longbottorn, D., 2012. New challenges for vaccination to prevent chlamydial abortion in sheep. Comparative Immunology Microbiology and Infectious Diseases 35, 271-276.

Faustino, C.R., Jennelle, C.S., Connolly, V., Davis, A.K., Swarthout, E.C., Dhondt, A.A., Cooch, E.G., 2004. Mycoplasma gallisepticum infection dynamics in a house finch population: seasonal variation in survival, encounter and transmission rate. Journal of Animal Ecology 73, 651-669.

George, T.L., Harrigan, R.J., LaManna, J.A., DeSante, D.F., Saracco, J.F., Smith, T.B., 2015. Persistent impacts of West Nile virus on North American bird populations. Proceedings of the National Academy of Sciences of the United States of America 112, 14290-14294.

Girjes, A.A., Carrick, F.N., Lavin, M.F., 1994. Remarkable sequence relatedness in the DNA encoding the major outer membrane protein of Chlamydia psittaci (Koala Type I) and Chlamydia pneumoniae. Gene 138, 139-142.

Girjes, A.A., Hugall, A.F., Timms, P., Lavin, M.F., 1988. Two distinct forms of Chlamydia psittaci associated with disease and infertility in Phascolarctos cinereus (koala). Infection and Immunity 56, 1897-1900.

Glassick, T., Giffard, P., Timms, P., 1996. Outer membrane protein 2 gene sequences indicate that Chlamydia pecorum and Chlamydia pneumoniae cause infections in koalas. Systematic and Applied Microbiology 19, 457-464.

Gordon, G., McGreevy, D.G., Lawrie, B.C., 1990. Koala populations in Queensland: major limiting factors, In Biology of the koala. eds A.K. Lee, K.A. Handasyde, G.D. Sanson, pp. 85-95. Surrey Beatty \& Sons, Sydney. 
Govendir, M., Hanger, J., Loader, J.J., Kimble, B., Griffith, J.E., Black, L.A., Krockenberger, M.B., Higgins, D.P., 2011. Plasma concentrations of chloramphenicol after subcutaneous administration to koalas (Phascolarctos cinereus) with chlamydiosis. Journal of Veterinary Pharmacology and Therapeutics 35, 147-154.

Griffith, J.E., Dhand, N.K., Krockenberger, M.B., Higgins, D.P., 2013. A retrospective study of admission trends of koalas to a rehabilitation facility over 30 years. Journal of Wildlife Diseases 49, 18-28.

Grogan, L.F., Berger, L., Rose, K., Grillo, V., Cashins, S.D., Skerratt, L.F., 2014. Surveillance for emerging biodiversity diseases of wildlife. Plos Pathogens 10, e1004015.

Hammerschlag, M.R., 1996. Diagnostic methods for intracellular pathogens. Clinical Microbiology and Infection 1, S3-S8.

Haydon, D.T., Cleaveland, S., Taylor, L.H., Laurenson, M.K., 2002. Identifying reservoirs of infection: A conceptual and practical challenge. Emerging Infectious Diseases 8, 1468-1473.

Hernandez-Sanchez, J., Brumm, J., Timms, P., Beagley, K.W., 2015. Vaccination of koalas with a prototype chlamydial vaccine is safe, does not increase the incidence of lymphoma-related disease and maybe associated with increased lifespan in captive koalas. Vaccine 33, 4459-4463.

Hirst, L.W., Brown, A.S., Kempster, R., Hall, J., Woolcock, J.B., 1992. Keratitis in free-ranging koalas (Phascolarctos cinereus) on Magnetic Island, Townsville. Journal of Wildlife Diseases 28, 424-427.

Hudson, P.J., Dobson, A.P., Newborn, D., 1998. Prevention of population cycles by parasite removal. Science 282, 2256-2258.

Hundloe, T., Hamilton, C., 1997. Koalas and Tourism: An Economic Evaluation. The Australia Institute, Lyneham, ACT, Australia.

Jackson, M., White, N., Giffard, P., Timms, P., 1999. Epizootiology of Chlamydia infections in two freerange koala populations. Veterinary Microbiology 65, 225-234.

Khan, S.A., Polkinghorne, A., Waugh, C., Hanger, J., Loader, J., Beagley, K., Timms, P., 2016. Humoral immune responses in koalas (Phascolarctos cinereus) either naturally infected with Chlamydia pecorum or following administration of a recombinant chlamydial major outer membrane protein vaccine. Vaccine 34, 775-782.

Lachish, S., McCallum, H., Mann, D., Pukk, C.E., Jones, M.E., 2010. Evaluation of Selective Culling of Infected Individuals to Control Tasmanian Devil Facial Tumor Disease. Conservation Biology 24, 841-851.

Lebreton, J.D., Pradel, R., 2002. Multistate recapture models: modelling incomplete individual histories. Journal of Applied Statistics 29, 353-369.

Lee, A.K., Martin, R.W., Handasyde, K.A., 1990. Experimental translocation of koalas to new habitat, In Biology of the koala. eds A.K. Lee, K.A. Handasyde, G.D. Sanson, pp. 299-312. Surrey Beatty \& Sons, Sydney.

Mackenzie, D.I., Nichols, J.D., Seamans, M.E., Gutierrez, R.J., 2009. Modeling species occurrence dynamics with multiple states and imperfect detection. Ecology 90, 823-835.

Marcus, A.D., Higgins, D.P., Gray, R., 2015. Ivermectin treatment of free-ranging endangered Australian sea lion (Neophoca cinerea) pups effect on hookworm and lice infection status, haematological parameters, growth, and survival. Parasitology Research 114, 2743-2755.

Markey, B., Wan, C., Hanger, J., Phillips, C., Timms, P., 2007. Use of quantitative real-time PCR to monitor the shedding and treatment of chlamydiae in the koala (Phascolarctos cinereus). Veterinary Microbiology 120, 334-342.

Martin, R., Handasyde, K., 1990. Population dynamics of the koala (Phascolarctos cinereus) in southeastern Australia. Biology of the koala, 75-84.

Martin, R.W., 1985a. Overbrowsing, and decline of a population of the koala, Phascolarctos cinereus, in Victoria. 2. Population condition. Australian Wildlife Research 12, 367-375.

Martin, R.W., 1985b. Overbrowsing, and decline of a population of the koala, Phascolarctos cinereus, in Victoria. 3. Population dynamics. Australian Wildlife Research 12, 377-385.

McAlpine, C., Lunney, D., Melzer, A., Menkhorst, P., Phillips, S., Phalen, D., Ellis, W., Foley, W., Baxter, G., de Villiers, D., Kavanagh, R., Adams-Hosking, C., Todd, C., Whisson, D., Molsher, R., Walter, M., Lawler, I., Close, R., 2015. Conserving koalas: A review of the contrasting regional trends, outlooks and policy challenges. Biological Conservation 192, 226-236.

McColl, K.A., Martin, R.W., Gleeson, L.J., Handasyde, K.A., Lee, A.K., 1984. Chlamydia infection and infertility in the female koala (Phascolarctos cinereus). Veterinary Record 115, 655-655. 
McInnes, L.M., Gillett, A., Hanger, J., Reid, S.A., Ryan, U.M., 2011. The potential impact of native Australian trypanosome infections on the health of koalas (Phascolarctos cinereus). Parasitology 138, 873-883.

McLean, N., Handasyde, K.A., 2006. Sexual maturity, factors affecting the breeding season and breeding in consecutive seasons in populations of overabundant Victorian koalas (Phascolarctos cinereus). Australian Journal of Zoology 54, 385-392.

Melzer, A., Carrick, F., Menkhorst, P., Lunney, D., John, B.S., 2000a. Overview, critical assessment, and conservation implications of koala distribution and abundance. Conservation Biology 14, 619-628.

Melzer, A., Carrick, F., Menkhorst, P., Lunney, D., St. John, B., 2000b. Overview, critical assessment, and conservation implications of koala distribution and abundance. Conservation Biology 14, 619-628.

Mitchell, C.M., Hovis, K.M., Bavoil, P.M., Myers, G.S.A., Carrasco, J.A., Timms, P., 2010. Comparison of koala LPCoLN and human strains of Chlamydia pneumoniae highlights extended genetic diversity in the species. Bmc Genomics 11.

Mitchell, P.J., Bilney, R., Martin, R.W., 1988. Population structure and reproductive status of koalas on Raymond Island, Victoria. Australian Wildlife Research 15, 511-514.

Murray, K.A., Skerratt, L.F., Speare, R., McCallum, H., 2009. Impact and dynamics of disease in species threatened by the amphibian chytrid fungus, Batrachochytrium dendrobatidis. Conservation Biology 23, 1242-1252.

Myers, G.S.A., Mathews, S.A., Eppinger, M., Mitchell, C., O'Brien, K.K., White, O.R., Benahmed, F., Brunham, R.C., Read, T.D., Ravel, J., Bavoil, P.M., Timms, P., 2009. Evidence that Human Chlamydia pneumoniae Was Zoonotically Acquired. Journal of Bacteriology 191, 7225-7233.

Obendorf, D.L., Handasyde, K.A., 1990. Pathology of chlamydial infection in the reproductive tract of the female koala (Phascolarctos cinereus), In Biology of the koala. eds A.K. Lee, K.A. Handasyde, G.D. Sanson, pp. 255-259. Surrey Beatty \& Sons, Sydney.

Patterson, J.L.S., Lynch, M., Anderson, G.A., Noormohammadi, A.H., Legione, A., Gilkerson, J.R., Devlin, J.M., 2015. The prevalence and clinical significance of chlamydial infection in island and mainland populations of Victorian koalas (Phascolarctos cinereus). Journal of Wildlife Diseases 51, 309-317.

Petticrew, M., 2001. Systematic reviews from astronomy to zoology: myths and misconceptions. British Medical Journal 322, 98-101.

Pickering, C., Byrne, J., 2014. The benefits of publishing systematic quantitative literature reviews for $\mathrm{PhD}$ candidates and other early-career researchers. Higher Education Research \& Development 33, 534548.

Polkinghorne, A., Hanger, J., Timms, P., 2013. Recent advances in understanding the biology, epidemiology and control of chlamydial infections in koalas. Veterinary Microbiology 165, 214-223.

Pradel, R., 1996. Utilization of capture-mark-recapture for the study of recruitment and population growth rate. Biometrics 52, 703-709.

Price, G.J., 2012. Chapter 9: Long-term trends in lineage ‘health’ of the Australian koala (Mammalia: Phascolarctidae): using paleo-diversity to prioritize species for conservation, In Paleontology in Ecology and Conservation. ed. J. Louys, pp. 171-192. Springer Earth System Sciences, SpringerVerlag, Berlin Heidelberg.

Rey-Ladino, J., Ross, A.G.P., Cripps, A.W., 2014. Immunity, immunopathology, and human vaccine development against sexually transmitted Chlamydia trachomatis. Human Vaccines \& Immunotherapeutics 10, 2664-2673.

Rhodes, J.R., Ng, C.F., de Villiers, D.L., Preece, H.J., McAlpine, C.A., Possingham, H.P., 2011. Using integrated population modelling to quantify the implications of multiple threatening processes for a rapidly declining population. Biological Conservation 144, 1081-1088.

Rochman, C.M., Browne, M.A., Underwood, A.J., van Franeker, J.A., Hompson, R.C.T., Amaral-Zettler, L.A., 2016. The ecological impacts of marine debris: unraveling the demonstrated evidence from what is perceived. Ecology 97, 302-312.

Scheele, B.C., Hunter, D.A., Banks, S.C., Pierson, J.C., Skerratt, L.F., Webb, R., Driscoll, D.A., 2016. High adult mortality in disease-challenged frog populations increases vulnerability to drought. Journal of Animal Ecology 85, 1453-1460.

Schneider, D.C., 2001. The rise of the concept of scale in ecology. Bioscience 51, 545-553. 
Skerratt, L.F., Berger, L., Hines, H.B., McDonald, K.R., Mendez, D., Speare, R., 2008. Survey protocol for detecting chytridiomycosis in all Australian frog populations. Diseases of Aquatic Organisms 80, 8594.

Skerratt, L.F., Berger, L., Speare, R., Cashins, S., McDonald, K.R., Phillott, A.D., Hines, H.B., Kenyon, N., 2007. Spread of chytridiomycosis has caused the rapid global decline and extinction of frogs. Ecohealth 4, 125-134.

Smith, K.F., Acevedo-Whitehouse, K., Pedersen, A.B., 2009. The role of infectious diseases in biological conservation. Animal Conservation 12, 1-12.

Stalder, K., Vaz, P.K., Gilkerson, J.R., Baker, R., Whiteley, P., Ficorilli, N., Tatarczuch, L., Portas, T., Skogvold, K., Anderson, G.A., Devlin, J.M., 2015. Prevalence and clinical significance of Herpesvirus infection in populations of australian marsupials. Plos One 10, 15.

Tarlinton, R., Meers, J., Hanger, J., Young, P., 2005. Real-time reverse transcriptase PCR for the endogenous koala retrovirus reveals an association between plasma viral load and neoplastic disease in koalas. Journal of General Virology 86, 783-787.

Thogmartin, W.E., King, R.A., McKann, P.C., Szymanski, J.A., Pruitt, L., 2012. Population-level impact of white-nose syndrome on the endangered Indiana bat. Journal of Mammalogy 93, 1086-1098.

Tompkins, D.M., Carver, S., Jones, M.E., Krkosek, M., Skerratt, L.F., 2015. Emerging infectious diseases of wildlife: a critical perspective. Trends in Parasitology 31, 149-159.

Vogelnest, L., Woods, R., CSIRO Publishing., 2008. Medicine of Australian mammals. CSIRO Publishing, Collingwood, Vic.

Waugh, C., Khan, S.A., Carver, S., Hanger, J., Loader, J., Polkinghorne, A., Beagley, K., Timms, P., 2016. A Prototype Recombinant-Protein Based Chlamydia pecorum Vaccine Results in Reduced Chlamydial Burden and Less Clinical Disease in Free-Ranging Koalas (Phascolarctos cinereus). Plos One 11.

White, N.A., Kunst, N.D., 1990. Aspects of the ecology of the koala in southeastern Queensland. Biology of the koala, 109-116.

Wobeser, G.A., 2007. Disease in Wild Animals: Investigation and Management, 2nd edition edn. Springer, New York. 